\section{P551 EXTRAGENITAL GONORRHEA POSITIVITY AMONG MEN WHO HAVE SEX WITH MEN - STD SURVEILLANCE NETWORK, 2015-2017}

${ }^{1}$ Winston Abara, ${ }^{2}$ Robert Kirkcaldy, ${ }^{3}$ Elizabeth Torrone, ${ }^{4}$ Eloisa Llata, ${ }^{5}$ Christina Schumacher, ${ }^{6} J u l i$ Carlos-Henderson, ${ }^{7}$ Dawn Ginzl, ${ }^{8}$ Roxanne Kerani, ${ }^{9}$ Kim Toevs, ${ }^{10}$ Preeti Pathela, ${ }^{11}$ Trang Nguyen, ${ }^{2}$ Kyle Bernstein. ${ }^{1}$ CDC, STD Prevention, Atlanta, USA; ${ }^{2}$ Centers for Disease Control and Prevention, Atlanta, USA; ${ }^{3}$ US Centers for Disease Control and Prevention, Division of STD Prevention, Atlanta, USA; ${ }^{4}$ Centers for Disease Control and Prevention, Division of STD Prevention, Atlanta, USA; ${ }^{5}$ Johns Hopkins School of Medicine, Baltimore, USA; ${ }^{6}$ Los Angeles County Department of Public Health, Los Angeles, USA; ${ }^{7}$ Minnesota Department of Health, Minnesota, USA; ${ }^{8}$ Public Health - Seattle and King County, HIVISTD Program, Seattle, USA; ${ }^{9}$ Multnomah County Health Department, Portland, USA; ${ }^{1}$ New York City Department of Health and Mental Hygiene, Bureau of Sexually Transmitted Infections, Long Island City, USA; ${ }^{11}$ San Francisco Department of Public Health, Arches Branch, Population Health Division, San Francisco, USA

\subsection{6/sextrans-2019-sti.626}

Background Extragenital gonorrhea (GC) is often asymptomatic, detected through screening of anatomic sites of exposure. Antimicrobial therapy with ceftriaxone plus azithromycin is the recommended GC treatment; monotherapy (azithromycin or doxycycline) is recommended for chlamydia (CT). In urethral CT-positive patients who are urethral GC-negative and not screened at extragenital sites, CT monotherapy may lead to GC undertreatment. We assessed urethral and extragenital GC positivity among MSM seeking care in STD clinics.

Methods Data were obtained from 30 STD clinics in 10 local health jurisdictions included in the STD Surveillance Network. Participants were adult MSM with $\geq 1$ clinic visit between 1/1/ 2015-12/31/2017. Data were analyzed by clinic visit. Using an inverse-variance random effects model to account for heterogeneity between jurisdictions, weighted positivity estimates and 95\% confidence intervals (CI) were calculated for overall, urethral, rectal, and pharyngeal GC among all MSM, and rectal and pharyngeal GC among MSM who were both urethral CTpositive and urethral GC-negative.

Results Of 100,613 GC and CT testing visits, overall GC positivity (positivity at any site) was $16.8 \%$ (95\% CI=14.6-19.0). By anatomic site, urethral GC positivity was $7.7 \% \quad(95 \%$ $\mathrm{CI}=5.9-9.5)$, rectal GC positivity was $12.5 \%(95 \% \mathrm{CI}=11.1-$ 13.9), and pharyngeal GC positivity was $9.2 \%(95 \% \mathrm{CI}=8.0-$ 10.4). Of 3,981 testing visits among urethral CT-positive and urethral GC-negative MSM who were tested at extragenital sites (rectum and/or pharynx), extragenital GC positivity was 10.1\% (95\% C.I=8.7-11.5), rectal GC positivity was 5.3\% (95\% CI $=4.3-6.3 \%)$, and pharyngeal GC positivity was $6.9 \%$ (95\% CI=5.7-8.1).

Conclusion Extragenital GC was fairly common among MSM. Without extragenital screening, $\sim 10 \%$ of extragenital gonococcal infections would have been missed and consequently undertreated in urethral CT-positive/urethral GC-negative MSM. In addition to potentially failing to cure GC and facilitating ongoing transmission, undertreatment with azithromycin $1 \mathrm{~g}$ or doxycycline could potentially select for resistance. These findings underscore the importance of extragenital screening in MSM per CDC guidelines.

Disclosure No significant relationships.

\section{P553 INITIATING A SEXUAL NETWORK STUDY AMONG MEN WHO HAVE SEX WITH MEN: A MIXED-METHODS PILOT STUDY}

${ }^{1}$ Janelle Ricks*, ${ }^{2}$ Morgan Spahnie, ${ }^{2}$ Sara Conroy, ${ }^{3}$ Dale Kiss, ${ }^{2}$ William Miller, ${ }^{4}$ Abigail Norris Turner. 'The Ohio State University, Division of Health Behavior and Health Promotion, Columbus, USA; ${ }^{2}$ The Ohio State University, Division of Epidemiology, Columbus, USA; ${ }^{3}$ The Ohio State University, Division of Epidemiology, Division of Health Behavior and Health Promotion, Columbus, USA; ${ }^{4}$ Ohio State University, Internal Medicine, Infectious Diseases, Columbus, USA

\subsection{6/sextrans-2019-sti.627}

Background In preparation for a prospective network study of men who have sex with men (MSM), we conducted a mixedmethods pilot study to examine feasibility and acceptability of several study components, including recruitment, data collection, and compensation.

Methods We conducted in-depth interviews (IDIs) with eight providers serving MSM and five focus group discussions (FGDs) with 34 MSM from four target MSM populations: young Black men, HIV-positive men, HIV-negative men on pre-exposure prophylaxis (PrEP), and men not engaged in medical care. We also conducted a 4-week pilot of a smartphone app for ecological momentary assessment (EMA) with 20 MSM (ongoing). EMA surveys were employed to capture daily data on anal and oral sex, consumption of alcohol and drugs, use of hook-up and social networking apps, and other behaviors. We used a brief exit survey to assess EMA app acceptability.

Results A major theme identified during IDIs and FGDs was the importance of developing trust and maintaining confidentiality during proposed respondent driven sampling (RDS) recruitment activities. A second FGD theme emphasized the importance of compensating participants appropriately for RDS and other study activities. All EMA participants reported being "completely comfortable" reporting their sexual behavior through the app. Most (67\%) preferred the app to in-person interviews. Several participants identified technical issues with the app, including not receiving push notifications and spontaneous app closure.

Conclusion IDIs and FGDs confirmed that developing trust and protecting participant confidentiality are critical for successful RDS recruitment. FGDs showed that MSM value their contributions to research and desire commensurate compensation. The EMA app was acceptable, despite technological challenges. It is feasible to use EMA to capture sexual behavior in this population. This mixed-methods pilot allowed for adjustments to the planned network study, including changes in compensation type and amount, troubleshooting technical issues, and modifications to the EMA survey.

Disclosure No significant relationships. 